\title{
Anticorpos antileucotoxina contra Actinobacillus actinomycetemcomitans em amostras de soro e saliva de pacientes com periodontite juvenil localizada ${ }^{\dagger}$
}

\section{Anti-leukotoxin antibodies against Actinobacillus actinomycetemcomitans in serum and saliva samples from patients with localized juvenile periodontitis}

\author{
Roberto Issamu NAKAGAWA* \\ Valéria H. GUAZELI-AMIN** \\ Mirian Marubayashi HIDALGO*** \\ Wilson TREVISAN Jr.**** \\ Eiko Nakagawa ITANO****
}

\begin{abstract}
NAKAGAWA, R. I.; GUAZELI-AMIN, V. H.; HIDALGO, M. M.; TREVISAN Jr., W.; ITANO, E. N. Anticorpos antileucotoxina contra Actinobacillus actinomycetemcomitans em amostras de soro e saliva de pacientes com periodontite juve-
\end{abstract} nil localizada. Pesqui Odontol Bras, v. 15, n. 1, p. 5-11, jan./mar. 2001.

\begin{abstract}
A leucotoxina de Actinobacillus actinomycetemcomitans é considerada seu principal fator de virulência com potencial de causar agressão às defesas do hospedeiro. No presente trabalho, foram analisados os niveis séricos e salivares de anticorpos antileucotoxina de A. actinomycetemcomitans em soros e salivas de pacientes com periodontite juvenil localizada (PJL) e controles saudáveis. Adicionalmente, foi realizada a análise de complexo imune (CI) nas amostras de saliva. Foram utilizados os métodos ELISA clássico com a leucotoxina obtida por gel filtração em Sephadex G-200 e ELISA de captura utilizando IgG de coelho anti- $A$. actinomycetemcomitans FDC Y4 leucotóxico adsorvido com uma cepa da mesma espécie, porém, não leucotóxica. Os resultados obtidos demonstraram níveis séricos de IgG significativamente mais elevados em pacientes com PJL em relação aos controles sadios, tanto por ELISA clássico como por ELISA de captura $(\mathrm{p}<0,05)$. No entanto, não foram observadas diferenças entre os níveis de IgG, IgA-S e CI nas salivas dos indivíduos examinados. Estes resultados sugerem que, embora A. actinomycetemcomitans apresente vários fatores de virulência que afetam a resposta imune do hospedeiro, ocorre resposta imune à leucotoxina nos pacientes com PJL. Esse aumento de IgG na circulação sangüinea pode contribuir na defesa do hospedeiro, limitando a lesão nas regiões periodontais amplamente colonizadas por A. actinomycetemcomitans.
\end{abstract}

UNITERMOS: Imunoglobulinas; Periodontite juvenil localizada; Actinobacillus actinomycetemcomitans; ELISA; Complexo antígeno-anticorpo.

\section{INTRODUÇÃO}

A periodontite juvenil localizada (PJL) é uma forma de periodontite caracterizada pela rápida progressão afetando, em especial, os primeiros molares e incisivos. Os pacientes apresentam anormalidades nos neutrófilos polimorfonucleares $(\mathrm{PMN})^{2,6,11}$ e freqüentemente a doença está associada à presença de Actinobacillus actinomycetemcomitans ${ }^{1,24}$.
Entre os vários fatores biologicamente ativos produzidos pelo A. actinomycetemcomitans que podem interferir no mecanismo de defesa do hospedeiro, existe uma leucotoxina que apresenta massa molecular de 115 a $180 \mathrm{kDa}^{23}$, capaz de destruir especificamente PMN e monócitos humanos ${ }^{6,20}$. Os produtos da lise celular liberados contribuem para a lesão tecidual e a diminuição dos monócitos acarreta alteração da resposta imune ao agente infeccioso ${ }^{10,18,19}$.

$\dagger$ Parte de Dissertação de Mestrado.

* Pós-graduando em Microbiologia; ** Professora Assistente; **** Professores Adjuntos - Universidade Estadual de Londrina UEL.

*** Professora Assistente - Universidade Estadual de Maringá - UEM.

Apoio financeiro da CPG/UEL e PROUNI/CCS/UEL. 
NAKAGAWA, R. I.; GUAZELI-AMIN, V. H.; HIDALGO, M. M.; TREVISAN Jr., W.; ITANO, E. N. Anticorpos antileucotoxina contra Actinobacillus actinomycetemcomitans em amostras de soro e saliva de pacientes com periodontite juvenil localizada. Pesqui Odontol Bras, v. 15, n. 1, p. 5-11, jan./mar. 2001.

Por outro lado, as propriedades leucotóxicas de A. actinomycetemcomitans podem ser moduladas pelo soro humano e animal ${ }^{14,23,25,26}$ e tem sido relatado por alguns autores aumento nos niveis de anticorpos específicos em pacientes com periodontites $^{13,26}$. Todavia, os dados encontrados na literatura são conflitantes ${ }^{7,17,21}$. Em amostras de saliva, HIDALGO et $a l^{7}$, corroborado por MONCADA et $a l^{15}$ e RUBIRA et al. ${ }^{16}$, não observaram diferenças entre pacientes e controles.

Considerando-se a importância da leucotoxina como um dos principais fatores de agressão ao hospedeiro e a capacidade de modulação pelo soro humano de sua atividade, o presente trabalho teve como objetivo verificar os niveis séricos e salivares de anticorpos antileucotoxina de A. actinomycetemcomitans em pacientes com PJL e controles. Adicionalmente, investigou-se a presença de complexos imunes (CI) contendo anticorpos específicos para este microrganismo na saliva.

\section{MATERIAL E MÉTODOS \\ Pacientes e indivíduos saudáveis}

Dez pacientes da Clínica de Periodontia, do Departamento de Medicina Oral e Odontologia Infantil da UEL, apresentando evidências clínicas e radiográficas de perda óssea alveolar localizada nos primeiros molares e incisivos permanentes com envolvimento de, no máximo, 14 dentes foram selecionados. Seis indivíduos pertenciam ao sexo feminino e 4 ao masculino, com idades entre 15 e 30 anos. Como controles, foram selecionados 10 indivíduos sadios sem evidência clínica ou radiográfica de doença periodontal e com idade e sexo semelhantes à população em estudo. Ninguém havia estado em tratamento com antibióticos durante os 3 meses anteriores à coleta de material e todos assinaram o termo de consentimento livre e esclarecido após informação. Este estudo foi aprovado pela Comissão de Bioética da Universidade Estadual de Londrina.

\section{Obtenção das amostras de saliva e soro}

A obtenção das amostras de saliva e soro foi realizada segundo o descrito por HIDALGO et $a l^{7}$ e, depois de aliquotadas, foram armazenadas a $-20^{\circ} \mathrm{C}$.

\section{Obtenção do extrato de}

\section{A. actinomycetemcomitans}

As amostras de A. actinomycetemcomitans FDC Y4 foram obtidas segundo o descrito por HIDALGO et $a l .^{8}$. Posteriormente, foi realizada a determinação de proteínas a partir do sobrenadante segundo método Folin descrito por LAYNE ${ }^{12}$ e os extratos foram aliquotados e armazenados a $-80^{\circ} \mathrm{C}$.

\section{Obtenção da leucotoxina de}

\section{A. actinomycetemcomitans FDC Y4}

Segundo GUAZELI-AMIN ${ }^{5}$, amostra de extrato sonicado da cepa $A$. actinomycetemcomitans FDC Y4 (1,4 mg de proteína/ $\mathrm{ml}$ ) foi aplicada em coluna de Sephadex G-200 $(2 \times 50 \mathrm{~cm})$ e as frações obtidas foram analisadas em espectrofotômetro a $280 \mathrm{~nm}$.

\section{Obtenção de IgG de coelho anti-extrato sonicado de $A$. actinomycetemcomitans FDC Y4}

Amostra de extrato sonicado de A. actinomycetemcomitans FDC Y4 $(150 \mu \mathrm{g} / \mathrm{ml})$ foi emulsificada com adjuvante incompleto de Freund e aplicada por via subcutânea em três doses, com intervalos de 15 dias, em coelhos jovens, machos, pesando aproximadamente $3,5 \mathrm{~kg}$. Após a sangria de prova e titulação do soro por imunodifusão dupla, realizou-se a sangria branca. A amostra de soro imune obtida foi passada na coluna de Sepharose-proteína $\mathrm{G}$ para purificação de IgG e, em seguida, adsorvida por $1 \mathrm{~h}$ a $37^{\circ} \mathrm{C}$ e $18 \mathrm{~h} \mathrm{a} 4^{\circ} \mathrm{C} \mathrm{v} / \mathrm{v}$, com $1,6 \mu \mathrm{g} / \mathrm{ml}$ de isolados de $A$. actinomycetemcomitans denominados de E8 e E10 previamente testados e considerados não leucotóxicos ${ }^{5}$.

\section{Adsorção do conjugado anti-IgG humana peroxidase}

Para adsorção do conjugado anti-IgG humana marcado com peroxidase, inicialmente foi obtido complexo IgG de coelho Sepharose-proteína G. A amostra de soro normal de coelho $(3 \mathrm{ml})$ foi passada na coluna de Sepharose-proteína G $(1,0 \mathrm{ml})$ e, após exaustivas lavagens com PBS, $10 \mathrm{ml}$ (1/1.000) de conjugado IgG de camundongo anti-IgG humana foram passados 3 vezes na mesma coluna. Esse conjugado adsorvido na diluição final aproximada de 1/2.000 foi utilizado para detecção de complexos imunes.

\section{Análise de IgG e IgA-S antileucotoxina de A. actinomycetemcomitans nas amostras de soro e saliva por ELISA clássico}

A placa de ELISA foi sensibilizada com a leucotoxina obtida através de gel filtração (fração I $8,7 \mu \mathrm{g} / \mathrm{ml}$ ) diluída em tampão carbonato-bicarbo- 
NAKAGAWA, R. I.; GUAZELI-AMIN, V. H.; HIDALGO, M. M.; TREVISAN Jr., W.; ITANO, E. N. Anticorpos antileucotoxina contra Actinobacillus actinomycetemcomitans em amostras de soro e saliva de pacientes com periodontite juvenil localizada. Pesqui Odontol Bras, v. 15, n. 1, p. 5-11, jan./mar. 2001.

nato $(0,1 \mathrm{~N})$ em um volume final de $200 \mu \mathrm{l}(\mathrm{v} / \mathrm{v})$ e incubada durante uma hora a $37^{\circ} \mathrm{C}$ seguida de uma noite a $4^{\circ} \mathrm{C}$. Após esse período, a placa foi bloqueada com leite desnatado $5 \%$ e Tween 20 a 0,5\% em PBS por $1 \mathrm{~h}$ seguida de quatro lavagens em PBS-Tween-leite. As amostras de soro $(1 / 200)$ ou saliva (1/10) dos grupos estudados foram incubadas por $1 \mathrm{~h}$. Posteriormente, a placa foi novamente lavada e incubada com conjugado anti-IgG humana peroxidase (1/2.000 - Sigma, EUA) por $1 \mathrm{~h}$. Para a análise de IgA-S, foi utilizada IgG de camundongo antipeça secretora de IgA humana (1/5.000 - Sigma, EUA) seguida de conjugado anti-IgG de camundongo peroxidase $(1 / 8.000-$ Sigma, EUA) e solução de ortofeniletilenodiamine di-hidrocloreto, sendo a leitura realizada a $492 \mathrm{~nm}$ (Multiskan).

\section{Análise de IgG antileucotoxina de A. actinomycetemcomitans nas amostras de soro e saliva por ELISA de captura}

Foi utilizada a mesma metodologia descrita para o ELISA clássico, porém, as placas de ELISA foram inicialmente tratadas com IgG de coelho anti-A. actinomycetemcomitans FDC Y4 adsorvida com cepa da mesma espécie não leucotóxica, para subseqüentemente serem sensibilizadas com o extrato sonicado de $A$. actinomycetemcomitans FDC Y4 $(50 \mu \mathrm{g} / \mathrm{ml})$.

\section{Análise de imunocomplexos em amostras de saliva}

Utilizando-se a mesma metodologia descrita para o ELISA clássico, as placas de ELISA foram sensibilizadas com a IgG de coelho anti- $A$. actinomycetemcomitans FDC Y4 adsorvida com cepa da mesma espécie não leucotóxica. Após bloqueio, as placas foram tratadas com amostras de saliva e a seguir com o conjugado IgG de camundongo anti-IgG humana-peroxidase adsorvida com IgG de coelho.

\section{Análise estatística}

A análise estatística foi realizada pelo testes ANOVA e $t$ de Student, considerando-se estatisticamente significantes os resultados com valores $\mathrm{p}<0,05$.

\section{RESULTADOS}

\section{Obtenção da leucotoxina de}

\section{A. actinomycetemcomitans}

A cromatografia do extrato sonicado de A. actinomycetemcomitans FDC Y4 em coluna de Sephadex G-200 resultou em dois picos com absorção a $280 \mathrm{~nm}$ (Gráfico 1). As amostras pertencentes ao primeiro pico foram agrupadas em um "pool" denominado fração I. Esta foi analisada quanto à sua pureza através de eletroforese em gel de poliacrilamida e quanto à sua atividade leucotóxica através da dosagem de lactato desidrogenase (LDH) citoplasmática liberada por células P3UI (mieloma de camundongo) com ela incubadas ${ }^{5}$.

\section{Análise de IgG sérica antileucotoxina}

Os resultados das médias de densidades ópticas (DO) a $492 \mathrm{~nm}$ dos ensaios com soros de pacientes com PJL $(\mathrm{n}=10)$ foram de 0,81 $\pm 0,17$ para ELISA clássico e 0,21 \pm 0,03 para ELISA de captu-



GRÁFICO 1 - Perfil espectrofotométrico das frações obtidas por cromatografia em coluna de Sephadex G-200 do extrato sonicado de A. actinomycetemcomitans FDC Y4. 
NAKAGAWA, R. I.; GUAZELI-AMIN, V. H.; HIDALGO, M. M.; TREVISAN Jr., W.; ITANO, E. N. Anticorpos antileucotoxina contra Actinobacillus actinomycetemcomitans em amostras de soro e saliva de pacientes com periodontite juvenil localizada. Pesqui Odontol

Bras, v. 15, n. 1, p. 5-11, jan./mar. 2001.

ra. Para os soros controles, a média foi de $0,53 \pm 0,16$ e $0,09 \pm 0,01$, respectivamente. As diferenças entre pacientes e controles foram estatisticamente significantes $(\mathrm{p}<0,05)$ nos dois testes (Gráfico 2).

\section{Análise de IgG salivar antileucotoxina}

Nos ensaios com salivas de pacientes com PJL $(\mathrm{n}=10)$, os resultados das médias de DO a $492 \mathrm{~nm}$ foram de 0,13 \pm 0,04 para ELISA clássico e 0,20 \pm 0,04 para ELISA de captura. Com as salivas dos controles saudáveis, a média foi de 0,09 $\pm 0,02$ e $0,18 \pm 0,04$, respectivamente. Nenhuma diferença foi observada entre pacientes e controles nos dois testes (Tabela 1).

\section{Análise de IgA-S salivar antileucotoxina}

A comparação entre as médias não apresentou diferença estatística, sendo de 0,10 $\pm 0,05$ para pacientes com PJL e 0,09 \pm 0,02 para controles (Tabela 1$)$.

\section{Análise de complexos imunes}

Como apresentado na Tabela 1 , as médias dos resultados da análise de CI foram de 0,09 \pm 0,03 e $0,09 \pm 0,02$, respectivamente para as salivas de pacientes com PJL e controles, não apresentando portanto nenhuma diferença estatisticamente significante.

\section{DISCUSSÃO}

Há na literatura controvérsias quanto aos níveis séricos de imunoglobulinas específicas para A. actinomycetemcomitans em pacientes com PJL. Enquanto alguns autores demonstraram um aumento nos níveis de anticorpos em pacientes com periodontites ${ }^{13,26}$, outros não encontraram diferenças $^{17,21}$. HIDALGO et al. ${ }^{7}$ sugeriram que a ausência de anticorpos poderia ser decorrente da falta de uma resposta imune específica devido à atividade da leucotoxina e/ou do(s) fator(es) supressor(es) presente(s) em $A$. actinomycetemcomitans, ou ainda devido ao mecanismo de tolerância já que $A$. actinomycetemcomitans pertence à microbiota normal da boca.

Um dos fatores de virulência de A. actinomycetemcomitans relacionado com a PJL é a leucotoxina. Para se certificar de que os anticorpos detecta-
GRÁFICO 2 - IgG sérica antileucotoxina de A. actinomycetemcomitans quantificados por ELISA clássico e de captura. (-) média aritmética. $\mathrm{PJL}=$ soros de pacientes com periodontite juvenil localizada. Controle $=$ soros de doadores saudáveis. PJL versus controle: $\mathrm{p}<0,05$.




NAKAGAWA, R. I.; GUAZELI-AMIN, V. H.; HIDALGO, M. M.; TREVISAN Jr., W.; ITANO, E. N. Anticorpos antileucotoxina contra Actinobacillus actinomycetemcomitans em amostras de soro e saliva de pacientes com periodontite juvenil localizada. Pesqui Odontol Bras, v. 15, n. 1, p. 5-11, jan./mar. 2001.

TABELA 1 - Imunoglobulinas antileucotoxina de A. actinomycetemcomitans e complexos imunes (CI) salivares quantificados por ELISA.

\begin{tabular}{l|c|c|c|c}
\hline \hline \multirow{2}{*}{} & \multicolumn{2}{|c|}{ ELISA clássico } & \multicolumn{2}{c}{ ELISA de captura } \\
\cline { 2 - 5 } & $\begin{array}{c}\text { PJL } \\
(\mathrm{n}=10)\end{array}$ & $\begin{array}{c}\text { Controle } \\
(\mathrm{n}=10)\end{array}$ & $\begin{array}{c}\text { PJL } \\
(\mathrm{n}=10)\end{array}$ & $\begin{array}{c}\text { Controle } \\
(\mathrm{n}=10)\end{array}$ \\
\hline IgG & $0,13^{*} \pm 0,04$ & $0,09 \pm 0,02$ & $0,20 \pm 0,04$ & $0,18 \pm 0,04$ \\
\hline IgA-S & $0,10 \pm 0,05$ & $0,09 \pm 0,02$ & - & - \\
\hline CI & $0,09 \pm 0,03$ & $0,09 \pm 0,02$ & - & - \\
\hline \hline
\end{tabular}

* Média \pm desvio-padrão da densidade óptica a $492 \mathrm{~nm}$. $\mathrm{PJL}=$ salivas de pacientes com periodontite juvenil localizada. Controle = salivas de doadores saudáveis. PJL versus Controle: $\mathrm{p}>0,05$.

dos em pacientes com PJL são de fato contra a leucotoxina, é necessária a sua utilização na forma purificada. No entanto, foi verificado que a atividade leucotóxica declina significativamente no processo de purificação, sendo observada atividade muito menor na fração obtida por gel filtração seguida de refracionamento em coluna de troca iôni$\mathrm{ca}^{5}$. A perda substancial da atividade biológica da leucotoxina também foi descrita por DiRIENZO ${ }^{3}$ em processos de purificação por cromatografia de afinidade utilizando anticorpos monoclonais. Por isso, no presente trabalho, foi utilizada a leucotoxina semipurificada em coluna de Sephadex G-200 (fração I).

Os resultados de ELISA clássico utilizando a leucotoxina demonstraram um nivel sérico de IgG significativamente maior em soros de pacientes com PJL em relação aos soros controles. Os resultados também demonstraram niveis de IgG significativamente maiores nos soros de pacientes com PJL, quando analisados por ELISA de captura. Possivelmente, por ser uma fração semipurificada, contendo antígenos mais específicos como a leucotoxina, foi possivel discriminar os grupos de pacientes e controles.

As IgGs do soro também podem estar presentes na saliva ${ }^{22}$. O aumento do nível sérico de IgG não foi acompanhado pelo aumento no nível salivar de IgG em pacientes com PJL, concordando com alguns relatos da literatura ${ }^{7,16}$.

Além de IgG, a IgA secretora produzida na mucosa é importante contra a colonização ou invasão de microrganismos por meio de sua ação neutrali$z^{2}$ te $^{9}$. Por isso, foi analisado, no presente trabalho, o nivel de IgA-S em amostras de saliva dos grupos estudados, mas não foi demonstrada dife- rença significativa. Este resultado poderia ser devido à formação de complexos imunes, conseqüentemente bloqueando o sítio combinatório da Ig. Assim, foi analisado adicionalmente o nível de CI nestas amostras de saliva.

Os resultados obtidos não demonstraram diferenças entre o nível de $\mathrm{CI}$ em amostras de saliva de pacientes com PJL e controles saudáveis. Por isso, a ausência de anticorpos específicos na saliva poderia ser explicada pela pequena amostragem analisada, diferenças de cepas regionais de A. actinomycetemcomitans, reações cruzadas em decorrência da utilização de leucotoxina semi-purificada, ausência de resposta imune localizada na mucosa oral, ou ainda pela degradação das Igs. Nesse sentido, GREGORY et al. ${ }^{4}$ encontraram, em sobrenadante de cultura de A. actinomycetemcomitans, enzimas proteolíticas que foram capazes de clivar in vitro IgG, IgA e IgM. Os autores também mostraram uma intensa degradação de IgG e IgA em amostras de fluido gengival de pacientes com PJL em comparação com indivíduos com periodonto saudável.

Os resultados obtidos sugerem que, embora $A$. actinomycetemcomitans apresente vários fatores de virulência que afetam a resposta imune do hospedeiro, resposta imune humoral à leucotoxina pode ser demonstrada na PJL. O aumento de IgG na circulação sangüínea pode contribuir na defesa do hospedeiro limitando a lesão às regiões periodontais amplamente colonizadas por A. actinomycetemcomitans.

\section{CONCLUSÕES}

1. Embora A. actinomycetemcomitans apresente atividade leucotóxica para monócitos humanos, ocorre resposta imune para a leucotoxina em pacientes com PJL, com aumento de IgG na circulação sangüínea.

2. Não foi observada diferença estatisticamente significante nos niveis salivares de IgG e IgA-S antileucotoxina em pacientes com PJL e indivíduos saudáveis e este resultado não se deve à formação de complexos imunes na saliva.

\section{AGRADECIMENTOS}

Os autores agradecem aos técnicos Mário Sumigawa Kaminami e Nilson de Jesus Carlos, pela excelente colaboração durante todo o desenvolvimento do trabalho e aos órgãos financiadores CPG/UEL e PROUNI/CCS/UEL. 
NAKAGAWA, R. I.; GUAZELI-AMIN, V. H.; HIDALGO, M. M.; TREVISAN Jr., W.; ITANO, E. N. Anticorpos antileucotoxina contra Actinobacillus actinomycetemcomitans em amostras de soro e saliva de pacientes com periodontite juvenil localizada. Pesqui Odontol Bras, v. 15, n. 1, p. 5-11, jan./mar. 2001.

NAKAGAWA, R. I.; GUAZELI-AMIN, V. H.; HIDALGO, M. M.; TREVISAN Jr., W.; ITANO, E. N. Anti-leukotoxin antibodies against Actinobacillus actinomycetemcomitans in serum and saliva samples from patients with localized juvenile periodontitis. Pesqui Odontol Bras, v. 15, n. 1, p. 5-11, jan./mar. 2001.

The leukotoxin produced by Actinobacillus actinomycetemcomitans is considered the major virulence factor with potential to cause damage to the host defenses. The present work analyzed the serumal and salivary levels of antibodies against the leukotoxin produced by A. Actinomycetemcomitans, in patients with Localized Juvenile Periodontitis (LJP) and in healthy controls. Additionally, analysis of the immune complex (IC) was carried out in saliva samples. The classic ELISA method, with leukotoxin obtained through Sephadex G-200 gel filtration, and the capture ELISA method, using rabbit anti-A. Actinomycetemcomitans (leucotoxic, FDC Y4, IgG) adsorbed with a non-leukotoxic strain of $A$. actinomycetemcomitans, were used. The results obtained demonstrated significantly higher serumal levels of IgG in patients with LJP, when they were compared with the healthy controls, both for the classic and capture ELISA methods ( $\mathrm{p}$ 0.05). However, no significant differences were observed between the salivary levels of IgG, SIgA and IC in the examined individuals. These results suggest that even though A. actinomycetemcomitans presents virulence factors that affect the immune response, there is immune response to leukotoxin in LJP patients. This increase of IgG in the blood stream might contribute to host defense, limiting the lesion to the periodontal regions already colonized by $A$. actinomycetemcomitans.

UNITERMS: Immunoglobulins; Localized juvenile periodontitis; Actinobacillus actinomycetemcomitans; Enzyme-linked immunosorbent assay; Antigen-antibody complex.

\section{REFERÊNCIAS BIBLIOGRÁFICAS}

1. ASIKAINEN, S.; JOUSIMIES-SOMER, H.; KANERVO, A.; SUMMANEN, P. Certain bacterial species and morphotypes in localized juvenile periodontitis and in matched controls. J Periodontol, v. 58, p. 224-230, 1987.

2. COGEN, R. B.; ROSEMAN, J. M.; AL-JOBURY, W. et al. Host factors in juvenile periodontitis. J Dent Res, v. 65, p. 394-399, 1986.

3. DiRIENZO, J. M. Application of monoclonal antibodies to the study of oral bacteria and their virulence factors. In: MACARIO, A. J. L.; MACARIO, E. C. Monoclonal antibodies against bacteria. New York : Academic Press, 1986. p. 249-293.

4. GREGORY, R. L.; KIM, D. E.; KINDLE, J. C; HOBBS, L. C. Imunoglobulin-degrading enzymes in localized juvenile periodontitis. J Periodont Res, v. 27, p. 176-183, 1992.

5. GUAZELI-AMIN, V. H. Análise da atividade leucotóxica de Actinobacillus actinomycetemcomitans e do efeito neutralizador de anticorpos antileucotoxina. Londrina, 1998. 67 p. Tese (Mestrado) - Universidade Estadual de Londrina.

6. HIDALGO, M. M.; AVILA-CAMPOS, M. J.; TREVISAN Jr., W. et al. Neutrophil chemotaxis and serum factor modulation in Brazilian periodontitis patients. Arch Med Res, v. 28, n. 4, p. 531-535, 1997.

7. HIDALGO, M. M. H.; ITANO, E. N.; NAKAGAWA, R. I. et al. Doença periodontal: estudo da resposta imune humoral. Rev Odontol Univ São Paulo, v. 12, n. 3, p. 207-213, 1998.

8. HIDALGO, M. M. H.; ITANO, E. N.; NISHIMURA, C. S. et al. Avaliação da resposta imune celular em pacientes com periodontite. Rev Odontol Univ São Paulo, v. 12, n. 2, p. 121-127, 1998.

9. KRAEHENBUHL, J. P.; NEUTRA, M. Molecular basis of immune protection of mucosal surfaces. Physiol Rev, v. 72, n. 4, p. 853-878, 1992.
10. KUBY, J. Immunology. 3. ed. New York : W. H. Freeman, 1997. $664 \mathrm{p}$.

11. LAVINE, W. S.; MADERAZO, E. G.; STOLMAN, J. et al. Impaired neutrophil chemotaxis in patients with juvenile and rapidly progressive periodontitis. J Periodont Res, v. 14, p. 10-19, 1979.

12. LAYNE, E. Spectrophotometric and turbinimetric methods for measuring protein. In: COLLOW, S. P.; KAPLAN, N. O. (eds.) Methods enzimology. New York : Academic Press, 1957. p. 447-454.

13. LISTGARTEN, M. A.; LAI, C. H.; EVIAN, C. I. Comparative antibody titers to Actinobacillus actinomycetemcomitans in juvenile periodontitis, chronic periodontitis and periodontally healthy subjects. J Clin Periodontol, v. 8, p. 155-164, 1981.

14. McARTHUR, W. P.; TSAI, C. C.; BAEHNI, P. C. et al. Leukotoxic effects of Actinobacillus actinomycetemcomitans. Modulation by serum components. J Periodont Res, v. 16, p. 159-170, 1981.

15. MONCADA, A. M.; ROCHA, R. S. S.; ROSA, O. P. S. et al. Determinação quantitativa da imunoglobulina G no tecido gengival de pacientes portadores de gengivite e periodontite através da imunodifusão radial simples. Estomat Cult, v. 16, n. 6, p. 9-13, 1986.

16. RUBIRA, I. R. F.; ROSA, O. P. S.; ROCHA, R. S. S.; REIS, M. A. Anticorpos séricos IgG anti-Actinobacillus actinomycetemcomitans Y4 e 29523 em indivíduos com diferentes condições periodontais. Rev Fac Odontol Bauru, v. 1, n. 1-4, p. 30-35, 1993.

17. SAITO, A.; HOSAKA, Y.; NAKAGAWA, T. Significance of serum antibody against surface antigens of Actinobacillus actinomycetemcomitans. Oral Microbiol Immunol, v. 8, p. 146-153, 1993.

18. SHENKER, B. J.; McARTHUR, W. P.; TSAI, C. C. Immune suppression induced by Actinobacillus actinomycetemcomitans. I - effects on human peripheral blood 
NAKAGAWA, R. I.; GUAZELI-AMIN, V. H.; HIDALGO, M. M.; TREVISAN Jr., W.; ITANO, E. N. Anticorpos antileucotoxina contra Actinobacillus actinomycetemcomitans em amostras de soro e saliva de pacientes com periodontite juvenil localizada. Pesqui Odontol Bras, v. 15, n. 1, p. 5-11, jan./mar. 2001.

lymphocyte response to mitogens and antigens. $\mathbf{J}$ Immunol, v. 128, p. 148-154, 1982.

19. SLOTS, J.; REYNOLDS, H. S.; GENCO, R. J. Actinobacillus actinomycetemcomitans in human periodontal disease: a cross-sectional microbiological investigation. Infect Immun, v. 29, p. 1013-1020, 1980.

20. TAICHMAN, N. S.; DEAN, R. T.; SANDERSON, C. J. Biochemical and morphological characterization of the killing of human monocytes by a leukotoxin derived from Actinobacillus actinomycetemcomitans. Infect Immun, v. 28, p. 258-268, 1980.

21. TAKAHASHI, K.; NAGAI, A.; SATOH, N. et al. Studies on the phenotypic and functional characterization of peripheral blood lymphocytes from patients with early-onset periodontitis. J Periodontol, v. 66, p. 391-396, 1995.

22. TENOVUO, J. Antimicrobial function of human saliva - how important is it for oral health? Acta Odontol Scand, v. 56, p. 250-256, 1998.

23. TSAI, C. C.; SHENKER, B. J.; DiRIENZO, J. M. et al. Extraction and isolation of a leukotoxin from Actinobacillus actinomycetemcomitans with polymyxin B. Infect Immun, v. 43, p. 700-705, 1984.

24. ZAMBON, J. J. Actinobacillus actinomycetemcomitans in human periodontal disease. J Clin Periodontol, v. 12, p. 1-20, 1985.

25. ZAMBON, J. J.; DeLUCA, C.; SLOTS, J.; GENCO, R. J. Studies of leukotoxin from Actinobacillus actinomycetemcomitans using the promyelocytic HL-60 cell line. Infect Immun, v. 40, p. 205-212, 1983.

26. ZAMBON, J. J.; SLOTS, J.; GENCO, R. J. Serology of oral Actinobacillus actinomycetemcomitans and serotype distribution in human periodontal disease. Infect Immun, v. 41, p. 19-27, 1983.

Recebido para publicação em 22/03/2000

Enviado para reformulação em 29/11/2000 Aceito para publicação em 23/01/2001
Sociedade Brasileira de Pesquisa Odontológica Av. Professor Lineu Prestes, 2227 - CEP 05508-900
Cidade Universitária - São Paulo - SP Tel./Fax: (0**1I) 38I8-7855 - E-mail.sbpqo@sbpqo.org.br 